\title{
Effects of body mass index on biomechanics of adult female foot
}

\begin{abstract}
Background \& Introduction: Adult obesity has been associated with a higher prevalence of musculoskeletal disorders, primarily affecting the lower limbs. Foot problems in obese adults are most important. Increased body weight is considered to cause overload of foot, which represents the interface between body and ground.
\end{abstract}

Aims \& Objectives: To study the effect of body weight on calcaneal eversion, angle of toe-out and gastrocnemius extensibility of adult female.

Materials \& Methods: Twenty female subjects at the age 20-30years were divided into 2 groups based on their Body Mass Index (BMI). Ten females with BMI 18.5-24.5 were assigned to Group A (Control) and rest ten were assigned to Group B (Overweight) having BMI between 25-29. A non- random convenient female sample was taken into the consideration. Subjects suffering from ligament injury, fracture, foot pain, limb length discrepancy were excluded from this study. Statistical analysis: Calcaneal eversion, gastrocnemius extensibility and angle of toe out in normal and overweight females were compared by applying unpaired t- test. P-value was set at 0.05 considered to be significant.

Results \& Discussion: The results showed no significant difference between the two groups in calcaneal eversion. Obesity can contribute to excessive pronation and foot pain. Gastrocnemius extensibility is less in overweight females $(p<0.001)$. This is because of excessive pronation which causes shortening of tendo-Achilles and instability at subtalar and midtarsal joint. The Angle of toe-out was found to be greater in overweight females $(\mathrm{p}<0.001)$. Overweight females have significant greater rear foot motion and foot angle values than normal weight females. Calcaneal eversion is greater in overweight females but it was statistically insignificant whereas angle of toe out was greater in overweight females which were highly significant. Gastrocnemius extensibility was less in overweight females when compared with normal subjects. Conclusion: We concluded that a high BMI could influence foot characteristics which may therefore predispose individuals to musculoskeletal pain.

Keywords: BMI, calcaneal eversion, gastrocnemius extensibility, angle of toe out
Volume 4 Issue I - 2017

\author{
Aparna Sarkar, Ashima Sawhney \\ Department of Physiology, Amity University, India
}

Correspondence: Aparna Sarkar, Associate Professor Physiology, Amity Physiotherapy College, Amity University, Uttar Pradesh, Noida, India,

Email asarkar@amity.edu,dr_aparna_sarkar@yahoo.co.in

Received: March 27, 2017 | Published: August 02, 2017

\section{Introduction}

Obesity is a major worldwide health problem where the body weight is more than $20 \%$ of the ideal. There are many long-term debilitating effects of obesity that may impair quality of life. These include cardiovascular disease, diabetes mellitus and various musculoskeletal disorders. ${ }^{1}$ Adult obesity has been associated with a higher prevalence of musculoskeletal disorders, primarily affecting the lower limbs. Musculoskeletal disorders, foot problems in obese adults are most important. This may be due to the increased stress placed on the feet through the need to bear excessive mass. Foot problems are frequent because the interface between body and ground is subjected to high stresses and load. The foot provides a stable support for the body, attenuates impact and rotational forces, provides sensory information, and combines flexibility and stability for propulsion of body. As increase in body weight is considered to cause overload of foot, which represents the interface between body and ground. This can induce various stress and strains during walking that can predispose to overuse injuries of lower limb. ${ }^{2,3}$

Gender and ethnic related Adult foot size is attained at about 14years of age in females and at about 16 in males, reflecting an earlier maturation in women. Women's feet have a considerably increased incidence of orthopedic problems. When foot length is identical, Asian feet have a wider forefoot width, a lower longitudinal arch, and more pronated feet. ${ }^{4}$ The rear foot acts as the central mechanism and a major weight bearing structure during stance. Authors suggested that the forefoot should be aligned perpendicular to the bisection of the calcaneus when the foot was in subtalar joint neutral. Any deviation from this position, either varus or valgus, was considered abnormal and could lead to abnormal motion and potential injury. They also suggested that individuals with a forefoot varus alignment might compensate through the mid tarsal and/or subtalar joint through excessive pronation. However, excessive pronation has been linked with injuries due muscle imbalance which disrupt normal lower limb alignment. This malalignment is thought to place undue stress and strain on the joints, ligaments and muscles.

Overweight and obesity are major health problems in many parts of the world and the incidence of these conditions is increasing. Among numerous other medical conditions, a high incidence of osteoarthritis, painful feet, and symptomatic complaints in the joints of the lower extremities are frequently reported for overweight persons. Plantar pressure analysis may provide additional insight into the etiology of pain and lower extremity complaints. The obese subjects show an increase in the forefoot width to foot length ratio, suggesting a 
broadening of the forefoot under increased weight loading conditions. In spite of the increased load bearing contact area of the foot with the ground, the obese men and women have substantially higher pressures under the heel, mid foot, and forefoot during standing. For obese the dynamic peak pressures during walking are also significantly increased under most foot areas, especially under the mid foot and across the metatarsal heads. In the static as well as the dynamic loading situation the highest pressure increases for the non-obese group are found under the longitudinal arch of the foot. Increases in pressure under the mid-foot are higher during standing for the obese women as compared to the obese men. ${ }^{4}$

Calcaneal eversion occurs in the frontal plane. It is one component of the tri-plane motion of pronation of the subtalar joint (STJ). Simultaneous with the eversion, a substantial amount of abduction occurs in the transverse plane at the STJ. The motion in the frontal and transverse planes at the STJ complements the ankle joint motion, which occurs primarily in the sagittal plane, to allow the rear foot to move in all three planes. The calcaneal eversion results from the striking the lateral aspect of the heel when we walk. The ground reaction force (GRF) forces the calcaneus to evert. Since the motion is produced by gravity and the GRF (motion given for free), the role of the muscles are to decelerate the motion. In this process, the muscles are lengthened and activated proprioceptively. The strain created in the muscles during the deceleration is transformed into a concentric motion-producing force. The calcaneal eversion that creates STJ pronation produces two important reactions in the body: one distal and one proximal. When the subtalar joint is pronated, the bones of the mid-tarsal joint (MTJ) are more mobile. During weight bearing this allows the foot to adapt to uneven surfaces and, more importantly, as the arch lowers certain muscles are lengthened (loaded) in order to become more powerful (e.g. peroneus longus). When the calcaneus begins to invert these processes are reversed in order to create a more stable foot at the time the muscles are "exploding." The proximal effects of calcaneal eversion are more significant. Because of the angle of the STJ axis, the frontal plane motion of the calcaneus creates transverse plane motion of the lower leg. The STJ is called a "torque converter" because it converts the frontal plane motion of the foot into transverse plane motion of the leg (and vice versa). This transverse plane motion of the lower leg often transfers to the femur, pelvis, and lumbar vertebrae. Because the STJ is tri-planar and all joints move in three planes, the calcaneal eversion during weight bearing produces tri-planar reactions in the knee, hip, and spinal joints. ${ }^{5}$

The foot provides dynamic stability for a person's entire body weight, while simultaneously maintaining flexibility for shock absorption and propulsion along uneven surfaces. As a result, there are unique biomechanical demands on the foot. One of the body's adaptations to these demands is through specialized movements in the foot, such as pronation. Most joint movements of the body are described in one of the three cardinal planes: sagittal, frontal or transverse. While there are foot motions such as dorsiflexion and plantar flexion that occur in these standard planes, most foot and ankle motion is not adequately described by these planes. More commonly, motion of the foot at the ankle is in a diagonal or oblique plane. This oblique plane motion involves movement through all three planes. For example, pronation is a diagonal plane movement of the foot around an oblique axis that combines the primary cardinal plane movements of dorsiflexion, eversion and abduction of the foot. Overpronation develops as a gradual biomechanical distortion. Several factors contribute to developing overpronation, including tibialis posterior weakness, ligament weakness, excess weight, pes planus (flat foot), genu valgum (knock knees), subtalar eversion, or other biomechanical distortions in the foot or ankle. Primary cause of over pronation is subtalar eversion, also called calcaneal valgus. When the calcaneus is everted, weight is forced onto the medial edge of the foot. Obesity can cause overpronation because the additional weight produces subtalar eversion and forces the longitudinal arch to collapse. Excessive pronation causes cumulative stress though the skeletal system and can lead to pain at the foot and ankle as well as proximally at the knee joint and in the low back. ${ }^{6}$

One of the common muscle imbalances that affect the ankle joint is a tight gastrocnemius-soleus. These muscles use $85 \%$ of their voluntary contraction during normal walking to help restrain the body's forward momentum by working eccentrically and concentrically. The passive extensibility is influenced by the size (mass) and length of muscle fibers, and the amount and arrangement of the connective tissues of the muscle belly. The resistance to passive lengthening is influenced by the readily adaptable amount of muscle tissue, including the contractile proteins and the non-contractile proteins of the sarcomere cytoskeletons. The relationship of adaptable changes in the muscle tissue and in the extracellular connective tissues remains unclear. Muscle length adaptations result from changes in the number of sarcomeres in series, which depend on the imposed length of muscles, not on the level of muscle activation and tension. Studies have suggested that optimal muscle function is probably achieved by increasing muscle length, length extensibility, passive elastic stiffness, mass and strength. ${ }^{7}$ Angle of toe out represents angle of foot placement or the FICK ANGLE and may be found by measuring the angle formed by each foot's line of progression and a line intersecting the centre of heel and second toe. It is approximately 120-180 from sagittal axis of the body, developing from 50 in children. The angle for men normally is about 70 of each foot at free speed walking. The degree of toe out decreases as speed of walking increases in normal men. ${ }^{8}$

Obesity leads to an increase in the occurrence of diverse diseases either independently or in association with other diseases and exacerbates disease progression as well as adversely affecting foot function. Increased weight on the feet significantly increases contact areas, with increased pressure on these areas leading to increased foot problems such as pain, vascular and neuropathic disease, deformity and joint mobility. ${ }^{9}$ Researcher suggested a link between higher body mass and flattening of the arches. Extra weight puts stress on the foot causing flattening of the arches..$^{10}$ In weight bearing, there is flatness of longitudinal arch, which is usually accompanied by out-toeing. ${ }^{11}$ It was noted that females had significantly more ROM in plantar flexion than their male counterparts. Authors studied that female between 17 and 30yrs of age had greater ROM in plantar flexion as well as dorsiflexion than males in same age groups. Research has show that significantly higher stiffness values at 100 of dorsiflexion in males compare to females. ${ }^{12}$ It was concluded that obesity has an excessive pronatory effect. ${ }^{13}$ The rear foot acts as the central mechanism and a major weight bearing structure during stance. ${ }^{14}$ It was suggested that the forefoot should be aligned perpendicular to the bisection of the calcaneus when the foot was in subtalar joint neutral. Any deviation from this position, either varus or valgus, was considered abnormal and could lead to abnormal motion and potential injury. It was also suggested that individuals with a forefoot varus alignment might compensate through the midtarsal and/or subtalar joint through excessive pronation. ${ }^{4}$ It was suggested that obesity can contribute to excessive pronation and foot pain. ${ }^{6}$ Excessive pronation causes shortening of tendo-achilles and instability at the subtalar and 
midtarsal joints. Excessive pronation is due to tight Tendo-Achilles (as stated above) or gastro- soleus. ${ }^{15,16}$ The compensation for reduced ankle dorsiflexion takes place at the subtalar and midtarsal joints in the form of excessive pronation. ${ }^{17}$ Foot placement angles may influence the amount of pronation exhibited. ${ }^{18}$

It was concluded that increased out-toeing increases pressure on the medial foot and provides mechanical force directed at the valgus foot. The in- toeing gait unloads the medial foot and increases the severity of the varus foot. ${ }^{19}$ An in- toeing position caused by malalignment of the fore foot in relation to rest of the foot is similar to a mild clubfoot, without equines or supination of the heel. As a matter of fact, there may be pronation of the heel along with the adduction. ${ }^{20}$ It was suggested that toe-out may present due to contracture of the gastrocnemius and soleus. ${ }^{21}$ Severely obese females have significantly greater rear foot motion and foot angle values than normal weight females ${ }^{22}$ which can lead to certain dysmorphism of foot especially flat foot. ${ }^{10}$

When the subtalar joint is pronated during weight-bearing activities, dorsiflexion can occur at the subtalar and midtarsal joints and at the talocrural joint, so increased subtalar pronation before heel-off can compensate for limited dorsiflexion at the talocrural joint. Therefore, when clinician's measure ankle joint dorsiflexion or prescribe gastrocnemius stretching exercises to increase ankle joint dorsiflexion, they often advocate maintaining the subtalar joint in a neutral or supinated position to direct the force to the talocrural joint rather than to the subtalar and midtarsal joints. Some investigators have suggested that the position of the subtalar joint influences goniometric measurements of ankle joint dorsiflexion, but little evidence has demonstrated the effect of subtalar position during gastrocnemius stretching on gains in ankle dorsiflexion range of motion (ROM). A central, rather than a peripheral, mechanism causes the increase in joint ROM after static stretching, and increased tolerance to stretching is the proposed central mechanism. If an increased tolerance to stretching resulted in increased range of ankle dorsiflexion, joint positioning may not have been as relevant as it would have been if mechanical changes occurred within the contractile or passive elements of the gastrocnemius muscle. ${ }^{23}$ The aims of the study was to compare the effect of weight on calcaneal eversion, angle of toe-out and gastrocnemius extensibility.

\section{Materials and methods}

Twenty female subjects at the age group 20-30years were divided into 2 groups based on their Body Mass Index (BMI). Ten females with BMI 18.5-24.5 were assigned to Group A(control). A further ten subjects with $\mathrm{BMI}=25-29$ were assigned to Group B (overweight). A Non- Random convenient sample design is chosen. No subjects were included in the study with the history of the following at least 6 months prior to the start of the study. Subjects who were suffering from congenital deformity, ligament injury, other soft tissue injury, fracture, limb length discrepancy were excluded from the study.

\section{Procedure}

A) The subject is chosen according to the inclusion criteria.

B) This will follow an assessment of the subject to check for presence of any exclusion criteria, if any of the points from the exclusion criteria are present then the subject should not be called for the research if he is not having any of the exclusion criteria then proceed to next step.

C) The subject should be told about the research and the procedure and he should fill the consent form.

\section{Measurement of calcaneal eversion}

Subtalar joint eversion was determined with subjects positioned prone with lower half of calf off the edge of plinth. Sliding calipers were used to identify mid points on the calf calcaneus and lines were drawn along the midlines on the posterior third of calf and calcaneus. Range of eversion was measured using a goniometer. The axis of standard goniometer was placed between the malleoli in the frontal plane. The stationery arm of goniometer was placed over the line on the posterior region of the calf and the movable arm was placed over the line on posterior calf. The calcaneus was passively everted to obtain subtalar joint range of motion (ROM).

\section{Measurement of gastrocnemius extensibility}

The subject was positioned in prone position and a marker was used for marking the fibular head, lateral malleolus, base of 5th metatarsal tuberosity and 5th metatarsal head. The stationary arm of the goniometer was placed along the long axis of fibula by using the mark on the fibular head and the lateral malleolus. The moving arm of the goniometer was then placed parallel to the lateral border of the foot by using the marks on the base and head of 5th metatarsal. The axis of the goniometer was kept on the lateral border of the foot. The zero position of dorsiflexion was defined as 90 between the long axis of fibula and the lateral border of the foot. All measurements were recorded as the subjects achieved maximum dorsiflexion.

\section{Measurement of angle of toe out}

A 3meter walkway was created using crepe paper. A chair was placed at the end of walkway to provide orientation during the ambulation trail. Water-soluble ink was applied to the plantar surface of the right foot. The subject was instructed to walk along the walkway. From the second footprint, three consecutive footprints were evaluated for angle of toe-out. The line parallel to the edge of paper represented the line of progression. The longitudinal axis of footprint was determined as the line drawn from bisecting the widest part of heel through the center of 2 nd toe. The angle between line of progression and longitudinal foot axis presented the angle of toe-out. The average of all 3 right foot prints was calculated.

\section{Statistical analysis}

To compare the values of calcaneal eversion, gastrocnemius extensibility and angle of toe out in normal and overweight females, unpaired t- test was performed for 20 subjects with age group 20-23years. To know the effect of stretching exercise on gastrocnemius extensibility for overweight females, paired t-test was performed for 10 subjects with age group 20-30years. 5\% level of probability was adopted as the level of statistical significance. The angles of both the limbs were measured, but here we considered the values of one limb as the difference was not significant between the two.

\section{Results}

Figure 1 showed comparison of $\mathrm{CE}$ in control and overweight females. It was observed that there was a decrease in CE angles in normal females as compared to overweight females on calculating $\mathrm{t}$-value. The result was significant at $\mathrm{p}<0.10$.

Figure 2 showed comparison of GE in normal and overweight females. It shows there is a decrease in GE angles in overweight females as compared to normal females on calculating t-value. The result was significant at $\mathrm{p}<0.001$.

Figure 3 shows comparison of ATO in normal and overweight females. It shows there is a decrease in ATO angles in normal females 
as compared to overweight females on calculating t-value. The result was significant at $\mathrm{p}<0.001$.

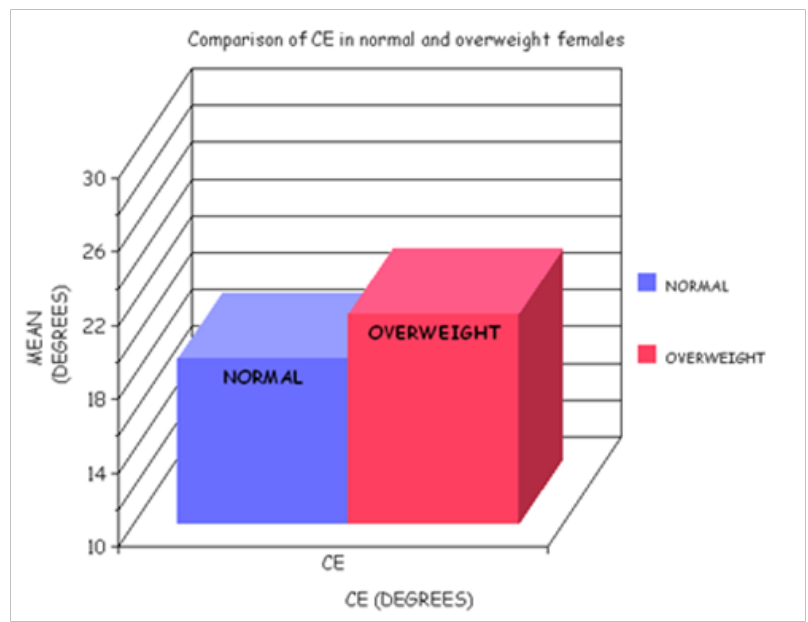

Figure I Comparison of Calcaneal Eversion (Ce) in control and overweight females.

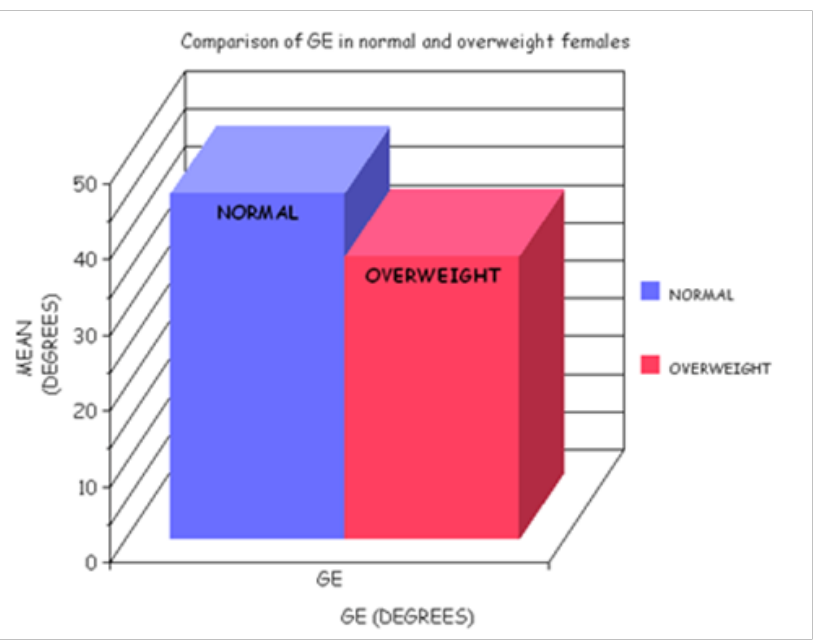

Figure 2 Comparison of gastrocnemius extensibilty (ge) in control and overweight females.

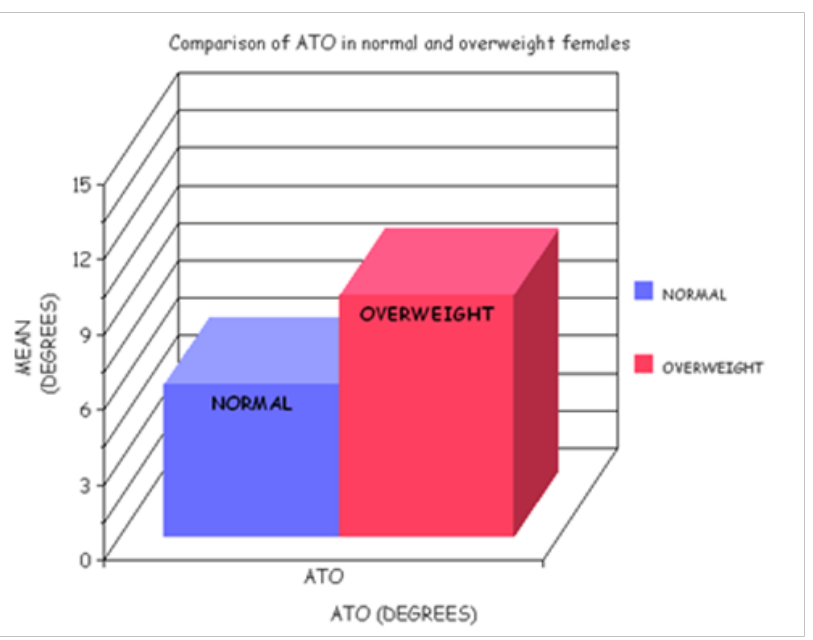

Figure 3 Comparison of Angle of Toe-Out (ATO) in control and overweight Females.

\section{Discussion}

The primary purpose of this study was to compare the effect of weight on calcaneal eversion, gastrocnemius extensibility and angle of toe out. Further aim was to know the effect of stretching exercise on gastrocnemius extensibility in overweight females. The results showed no significant difference between the two groups in calcaneal eversion. These findings are in contrast to Steven et al., ${ }^{6}$ who suggested that obesity can contribute to excessive pronation and foot pain. According to the results, Gastrocnemius extensibility is less in overweight females. This is supported by Harris \& Beath ${ }^{15}$ stating that the cause can be excessive pronation which causes shortening of tendo-Achilles and instability at subtalar and midtarsal joint.

The Angle of toe-out is found to be greater in overweight females in this study. It was also concluded by Messier SP et al.,22 that overweight females have significant greater rear foot motion and foot angle values than normal weight females. Kendall et al. ${ }^{11}$ concluded that in a weight bearing position, there is flatness of longitudinal arch that is usually accompanied by out toeing (i.e. increased ATO). An out toe position was found in subjects with a tight gastrocnemius. This result is found in overweight because toeing out in walking may result from tightness of tendo Achilles. Stretching exercise increased the gastrocnemius extensibility significantly in overweight females. Worrell et al, who reported increases in ankle dorsiflexion after gastrocnemius stretching with the subtalar joint positioned in either supination or pronation. ${ }^{23}$ The hypothesis proves that increased weight has an effect on calcaneal eversion, angle of toe-out and gastrocnemius extensibility. This is because extra weight puts stress on the foot causing flattening of the arches.

\section{Conclusion}

Considering the data attained in the present study, calcaneal eversion is greater in overweight females but is statistically insignificant whereas angle of toe out is greater in overweight females with a high level of significance. Gastrocnemius extensibility is less in overweight females when compared with normal subjects. Thus, the null hypothesis ( $\mathrm{H} 0)$ is rejected and the alternate hypothesis $(\mathrm{H} 1)$ is accepted. The study revealed that stretching exercise increases the gastrocnemius extensibility in overweight females thereby resulting in acceptance of the alternate hypothesis (H1) and rejection of null hypothesis (H0).The findings of this study show that a higher BMI can influence foot characteristics which may therefore predispose individuals to musculoskeletal pain.

\section{Acknowledgements}

None.

\section{Conflict of interest}

Author declares that there is no conflict of interest.

\section{References}

1. Riddiford Harland DL, Steele JR, Storlien LH. Does obesity influence foot structure in prepubescent children? Int J Obes Relat Metab Disord. 2000;24(5):541-544.

2. Vicenzino B, Fielding J, Howard, R, et al. An Investigation of the antipronation effect of two taping methods after application and exercise. Gait Posture. 1997;5(1):1-5.

3. Doxey GE. Calcaneal pain: A review of various disorders. $J$ Orthop Sports Phys Ther. 1987;9(1):25-32. 
4. Hennig EM. Invited Wei Lun Public Lecture: The Human Foot During Locomotion - Applied Research for Footwear. Hong Kong: The Chinese University of Hong Kong; 2002.

5. Buchanan KR, Davis I. The Relationship between forefoot, midfoot, and rearfoot static alignment in pain-free individuals. J Orthop Sports Phys Ther. 2005;35(9):559-566.

6. Stovitz SD, Coetzee JC. Hyperpronation and foot pain: steps toward pain-free feet. Phys Sportsmed. 2004;32(8):19-26.

7. Gajdosik RL. Passive extensibility of skeletal muscle: review of the literature with clinical implications. Clin Biomech (Bristol, Avon). 2001;16(2):87-101.

8. Cynthia C Norkin, Joyce White D. Measurement of Joint Motion: A Guide to Goniometry. 5th ed. USA: FA Davis Company; 1995. 592 p.

9. Jelinek HF, Fox D. Foot health and elevated body mass index. The Foot and Ankle Online Journal. 2009;2(8):4.

10. Carine HM van Schie, Boulton AJM. The effect of arch height and body mass on plantar pressure wounds. Medscape. 2000;12(4):88-95.

11. Kendall FP, McCreary EK, Provance PG. Muscles: Testing and Function. Br J Sports Med. 1984;18(1):25.

12. Cynthia C Norkin, Joyce white D. Measurement of joint motion. 3rd ed. Philadelphia: FA Davis; 1995. 248p.

13. Hall CM, Thein Broody L. Therapeutic Exercises-Moving Towards Function. 2nd ed. Philadelphia: Lippincott and Wilkins; 1999. 787 p.
14. Karas A, Hoy J. Compensatory mid foot dorsiflexion in individuals with heel cord tightness. J Prosthetics Orthotics. 2002;14(2):82-93.

15. Harris RI, Beath T. Hypermobile flatfoot with short tendo achilles. $J$ Bone Joint Surg. 1948;30A(1):116-140.

16. Charrette M. Orthotic support for overweight and obese patients. The Chiropractic Journal. 2002;14(2):82-93.

17. Buchanan KR, Davis I. The relationship between forefoot, midfoot and rear foot static alignment in pain-free individuals. $J$ Orthop Sports Phys Ther. 2005;35(9):559-566.

18. Kernozek TW, Ricard MD. Foot placement angle and arch type: effect on rear foot motion. Arch Phys Med Rehabil. 1990;71(12):988-991.

19. Chang WN, Tsirikos AI, Miller F, et al. Impact of changing FPA on foot pressure measurement in children with neuromuscular diseases. Gait Posture. 2004;20(1):14-19.

20. Brown LP, Yavorsky P. Locomotor biomechanics and pathomechanics: A review. J Orthop Sports Phys Ther. 1987;9(1):3-10.

21. Magee DJ. Orthopaedic Physical Assessment. 6th ed. Philadelphia: WB Saunders; 2014. 1184 p.

22. Messier SP, Davies AB, Moore DT, et al. Severe obesity: Effects on foot mechanics during walking. Foot Ankle Int. 1994;15(1):29-34.

23. Johanson M, Baer J, Hovermale H, et al. Subtalar joint position during gastrocnemius stretching and ankle dorsiflexion range of motion. J Athl Train. 2008;43(2):172-178. 